KYUNGPOOK Math. J. 55(2015), 21-28

http://dx.doi.org/10.5666/KMJ.2015.55.1.21

pISSN 1225-6951 eISSN 0454-8124

(c) Kyungpook Mathematical Journal

\title{
A Note on Skew-commuting Automorphisms in Prime Rings
}

\author{
NADEem uR Rehman* AND TARANnum Bano \\ Department of Mathematics, Aligarh Muslim University, Aligarh, 202002, India \\ e-mail : rehman100@gmail.com and tarannumdlw@gmail.com
}

Abstract. Let $R$ be a prime ring with center $Z, I$ a nonzero ideal of $R$, and $\sigma$ a nontrivial automorphism of $R$ such that $\left\{(x \circ y)^{\sigma}-(x \circ y)\right\}^{n} \in Z$ for all $x, y \in I$. If either $\operatorname{char}(R)>n$ or char $(R)=0$, then $R$ satisfies $s_{4}$, the standard identity in 4 variables.

\section{Introduction}

Throughout this paper, unless specifically stated, $R$ will be an associative ring, $Z$ the center of $R, Q$ its two sided Martindale quotient ring and $C$ its extended centroid. We refer the reader to [3] for the definitions and related properties of these objects. For $x, y \in R$, we denote $[x, y]=x y-y x$, the commutator of $x$ and $y$ and $x \circ y=x y+y x$, the anti-commutator (skew-commutator) of $x$ and $y$. Recall that a ring $R$ is prime if for any $a, b \in R, a R b=0$ implies that $a=0$ or $b=0$. Let $n \geq 1$ be a fixed integer and $s_{4}$ be the standard identity in 4 variables. For a subset $S$ of $R$, a mapping $f: S \rightarrow R$ is called commuting (centralizing) if $[f(x), x]=0$ (resp. $[f(x), x] \in Z)$ for all $x \in S$. A mapping $f: S \rightarrow R$ is called skew-commuting (skewcentralizing) if $f(x) \circ x=0$ (resp. $f(x) \circ x \in Z$ ) holds for all $x \in S$. The study of commuting and centralizing mappings goes back to 1955 when Divinsky [10] proved that a simple artinian ring is commutative if it has a commuting automorphism different from the identity mapping. Two year later Posner [17] showed that a prime ring must be commutative if it possesses a nonzero centralizing derivation. In 1970 Luh [14] generalized Divinsky's result to prime rings. Later, Mayne [16] obtained the analogous result of Posner for non identity centralizing automorphisms. Similar results extended to the case of left ideal by Bell and Martindale [4] and Lanski [13].

In [5] Brešar obtained a characterization of commuting additive mappings on

\footnotetext{
* Corresponding Author.

Received February 15, 2014; accepted April 22, 2014.

2010 Mathematics Subject Classification: 16N60, 16W20.

Key words and phrases: Prime ring, Ideal, Automorphism.
} 
prime rings. Based on this result, Brešar initiated the study of functional identities. We refer the reader to Brešar [7] for an introductory account on functional identities and their applications. In [6], Brešar proved that there are no nonzero skew-commuting additive mappings on a 2 - torsion free semiprime rings. In other words, if $R$ is a 2-torsion free semiprime ring and $f: R \rightarrow R$, an additive mapping such that $f(x) \circ x=0$, for all $x \in R$, then $f=0$.

In [2] Ashraf and Rehman proved that, If $R$ is a prime ring, $I$ a nonzero ideal of $R$ and $d$ is a derivation of $R$ such that $d(x \circ y)-(x \circ y)=0$ for all $x, y \in I$, then $R$ is commutative. Further in [1] Argac and Inceboz generalized this result, and obtained the following result, if $R$ is a prime ring, $I$ a nonzero ideal of $R$ and $d$ is a derivation of $R, n$ is a fixed positive integer and $(d(x \circ y))^{n}-(x \circ y)=0$ or is central for all $x, y \in I$, then the ring is commutative. The present paper is motivated by the previous results and we here continue this line of investigation by examining what happens in case the derivation is replaced by automorphism. Explicitly we shall prove the following theorem:

Theorem A. Let $R$ be a prime ring with center $Z, I$ a nonzero ideal of $R$, and $\sigma$ a nontrivial automorphism of $R$ such that $\left\{(x \circ y)^{\sigma}-(x \circ y)\right\}^{n} \in Z$ for all $x, y \in I$. If either $\operatorname{char}(R)>\mathrm{n}$ or char $(R)=0$, then $R$ satisfies $s_{4}$, the standard identity in 4 variables.

\section{Main Result}

We begin our discussion with the following well-known results.

Lemma 2.1.([18, Lemma 2.1]) Let $R$ be a prime ring with the extended centroid $C$. Then the following conditions are equivalent:

(i) $\operatorname{dim}_{C} R C \leq 4$;

(ii) $R$ satisfies $s_{4}$, the standard identity in 4 variables;

(iii) $R$ is commutative or $R$ embeds in $M_{2}(F)$ for $F$ a field;

(iv) $R$ is algebraic of bounded degree 2 over $C$;

(v) $R$ satisfies $\left[\left[x^{2}, y\right],[x, y]\right]$.

An automorphism $\sigma$ of $R$ is called $Q$-inner if there exists an invertible element $b \in Q$ such that $x^{\sigma}=b x b^{-1}$ for all $x \in R$. Otherwise, $\sigma$ is called outer. We denote by $A$ the group of all automorphisms of $R$ and by $G_{i}$ the group consisting of all $Q$-inner automorphisms of $R$. Recall that a subset $S$ of $A$ is said to be independent (modulo $G_{i}$ ) if for any $g_{1}, g_{2} \in S, g_{1} g_{2}^{-1} \in G i$ implies $g_{1}=g_{2}$. For instance, if $g$ is an outer automorphism of $R$, then 1 and $g$ are independent (modulo $G_{i}$ ). The following useful result is due to Chuang [9]. We only state a special case for our proofs in the sequel and refer its proof to that of Chuang [9]. 
Lemma 2.2. ([9, Theorem 3]) Suppose that $R$ is a prime ring and $S$ an independent subset of A modulo Gi. Let $\phi=\psi\left(x_{i}{ }^{g_{j}}\right)=0$ be a generalized identity with automorphisms of $R$ reduced with respect to $S$. If, for all $x_{i} \in X, g_{j} \in S$, the $x_{i}{ }^{g_{i}}-$ word degree of $\phi=\psi\left(x_{i}{ }^{g_{j}}\right)$ is strictly less than $\operatorname{char}(R)$ when $\operatorname{char}(R) \neq 0$, then $\psi\left(z_{i j}\right)=0$ is also a generalized polynomial identity of $R$.

We begin our discussion with the following result, which is essential for developing the proof of our main theorem.

Theorem 2.1. Let $R$ be a prime ring, $I$ a nonzero ideal of $R$, and $\sigma$ a nontrivial automorphism of $R$ such that $\left\{(x \circ y)^{\sigma}-(x \circ y)\right\}^{n}=0$ for all $x, y \in I, n$ be fixed positive integer. If either $\operatorname{char}(R)>n$ or char $(R)=0$, then $R$ satisfies $s_{4}$.

Proof. We assume that $\operatorname{dim}_{C} R C>4$. By assumption, we get that

$$
\left\{(x \circ y)^{\sigma}-(x \circ y)\right\}^{n}=0 \quad \text { for all } x, y \in I .
$$

Since $\sigma$ is an automorphism of $\mathrm{R}$ such that $\sigma \neq 1$, we have

$$
\left\{\left(x^{\sigma} \circ y^{\sigma}\right)-(x \circ y)\right\}^{n}=0 \quad \text { for all } x, y \in I .
$$

If $\sigma$ is an $Q$-outer automorphism of $R$, by Chuang ([9], Main Theorem), $R$ also satisfies identity (2.1). Since either char $(R)>n$ or char $(R)=0$, it follows from Lemma 2.2 that

$$
\{(z \circ w)-(x \circ y)\}^{n}=0 \quad \text { for all } x, y, z, w \in R .
$$

It is well known that there exists a field $F$ such that $R$ and $F_{m}$ satisfy the same polynomial identities (see Jacobson, [12], p. 57, p. 89). Let $e_{i j}$ be a matrix unit with 1 in $(i, j)$-entry and zero elsewhere. Suppose $m \geq 2$, if we choose $z=e_{11}, w=$ $e_{22}, x=e_{12}, y=e_{21}$, then we get a contradiction:

$$
\begin{aligned}
0 & =\left\{\left(e_{11} \circ e_{22}\right)-\left(e_{12} \circ e_{21}\right)\right\}^{n} \\
& =(-1)^{n} e_{11}+(-1)^{n} e_{22} \neq 0 .
\end{aligned}
$$

This forces $\mathrm{m}=1$, that is $R$ is commutative, a contradiction.

We now assume that $\sigma$ is $Q$-inner. So there exists an invertible element $b \in Q$ such that $x^{\sigma}=b x b^{-1}$ for all $x \in R$. We note that $b \notin C$ since $\sigma \neq 1$. By a theorem of Chuang [8], $Q$ and $I$ satisfy the same generalized polynomial identities (GPI's). From (2.1) we see that

$$
\left\{b(x \circ y) b^{-1}-(x \circ y)\right\}^{n}=0 \quad \text { for all } x, y \in Q .
$$

Since $Q$ remains prime by the primeness of $R$, replacing $R$ by $Q$ we may assume that $b \in R$ and $C$ is just the center of $R$. Note that $R$ is a centrally closed prime $C$-algebra in the present situation (Erickson et al., [11]), i.e., $R C=R$. 
Since $b \notin C$, it is obvious that (2.3) is a nontrivial GPI on $R$. By Martindale's theorem [15], $R$ is a strongly primitive ring. Let $V_{R}$ be a faithful irreducible right $R$-module with commuting division ring $D$. By the density theorem, $R$ acts densely on ${ }_{D} V$.

For any given $v \in V$, we claim that $v$ and $v b$ are $D$-dependent. If $v b=0$, then $v$ and $v b$ are $D$-dependent and we are done in this case. Suppose that $v b \neq 0, v$ and $v b$ are $D$-independent. We consider the following two cases.

Case 1. Assume that $v, v b, v b^{-1}$ are $D$-independent, by the density of $R$, there exist $x, y \in R$ such that

$$
\begin{aligned}
& v x=0, \quad v b x=v b^{-1}, \quad v b^{-1} x=0, \\
& v y=0, \quad v b y=0, \quad v b^{-1} y=v b .
\end{aligned}
$$

From (2.3), we get a contradiction:

$$
0=v\left\{b(x \circ y) b^{-1}-(x \circ y)\right\}^{n}=v \neq 0 .
$$

Case 2. Otherwise, $v, v b, v b^{-1}$ are $D$-dependent. Since $v$ and $v b$ are $D$-independent, we have $v b^{-1}=d_{1} v+d_{2} v b$, for some $d_{1}, d_{2} \in D$. Moreover, we claim that $d_{2} \neq 0$. Indeed, if $d_{2}=0$, then $v b^{-1}=d_{1} v$ and $v=d_{1} v b$, a contradiction. By the density of $R$ there exist $x, y \in R$ such that

$$
\begin{array}{ll}
v x=v, & v b x=0 \\
v y=-v, & v b y=v b
\end{array}
$$

and so from (2.3), we get a contradiction:

$$
0=v\left\{b(x \circ y) b^{-1}-(x \circ y)\right\}^{n}=v 2^{n} \neq 0 .
$$

From the above, we have proven that $v$ and $v b$ are $D$-dependent, i.e., $v b=\alpha(v) v$ for all $v \in V$, where $\alpha(v) \in D$ depends on $v \in V$. In fact, it is easy to check that $\alpha(v)$ is independent of the choice of $v \in V$. i.e., there exists $\lambda \in D$ such that $v b=\lambda v$ for all $v \in V$. We claim $\lambda \in Z(D)$, the center of $D$. Indeed, for any $\beta \in D$,

$$
(\beta v) b=\lambda(\beta v)=(\lambda \beta) v
$$

and on the other hand

$$
(\beta v) b=\beta(v b)=\beta(\lambda v)=(\beta \lambda) v
$$

From the above two expression we can get $\lambda \beta=\beta \lambda$, which implies $\lambda \in Z(D)$. So $b \in C$, a contradiction. Thus $\operatorname{dim}_{C} R C \leq 4$. By Lemma 2.1, R satisfies $s_{4}$. This completes the proof. 
Now, we are well equipped to prove our main result.

Theorem 2.2. Let $R$ be a prime ring, $I$ a nonzero ideal of $R$, and $\sigma$ a nontrivial automorphism of $R$ such that $\left\{(x \circ y)^{\sigma}-(x \circ y)\right\}^{n} \in Z$ for all $x, y \in I$, n be fixed positive integer. If either $\operatorname{char}(R)>n$ or char $(R)=0$, then $R$ satisfies $s_{4}$.

Proof. We assume that $\operatorname{dim}_{C} R C>4$. By assumption we get that,

$$
\left[\left\{(x \circ y)^{\sigma}-(x \circ y)\right\}^{n}, r\right]=0 \quad \text { for all } x, y, r \in I .
$$

Since $\sigma$ is an automorphism of $R$ with $\sigma \neq 1$, an identity map, we have

$$
\left[\left\{\left(x^{\sigma} \circ y^{\sigma}\right)-(x \circ y)\right\}^{n}, r\right]=0 \quad \text { for all } x, y, r \in I .
$$

If $\sigma$ is an $Q$-outer automorphism of $R$, by Chuang ([9], Main Theorem), $R$ also satisfies identity (2.4). Since either char $(R)>\mathrm{n}$ or char $(R)=0$, it follows from Lemma 2.2 that

$$
\left[\{(z \circ w)-(x \circ y)\}^{n}, r\right]=0 \quad \text { for all } \quad x, y, r, z, w \in R .
$$

It is well known that there exists a field $F$ such that $R$ and $F_{m}$ satisfy the same polynomial identities (see Jacobson, [12], p. 57, p. 89). Let $e_{i j}$ be a matrix unit with 1 in $(i, j)$-entry and zero elsewhere. Since $\operatorname{dim}_{C} R C>4$, we see that $m>2$. If we choose $z=e_{11}, w=e_{22}, x=e_{12}, y=e_{21}, r=e_{13}$, then by (2.5) we get a contradiction as follows

$$
0=\left[\left\{\left(e_{11} \circ e_{22}\right)-\left(e_{12} \circ e_{21}\right)\right\}^{n}, e_{13}\right]=(-1)^{n} e_{13} \neq 0 .
$$

We now assume that $\sigma$ is $Q$-inner. So there exists an invertible element $b \in Q$ such that $x^{\sigma}=b x b^{-1}$ forall $x \in R$. Since $\sigma \neq 1$, we see that $b \notin C$. By a theorem of Chuang [8], $Q$ and $I$ satisfy the same generalized polynomial identities. It follows from (2.4) that

$$
\left[\left\{b(x \circ y) b^{-1}-(x \circ y)\right\}^{n}, r\right]=0 \quad \text { for all } \quad x, y, r \in Q .
$$

Since $b \notin C$, it is obvious that (2.6) is a nontrivial GPI on $Q$. By Martindale's theorem [15], $Q$ is a strongly primitive ring. Let $V_{Q}$ be a faithful irreducible right $Q$-module with commuting ring $D=\operatorname{End}\left(V_{Q}\right)$, a finite dimensional division algebra over $C$. By the density theorem, $Q$ acts densely on ${ }_{D} V$.

If $\operatorname{dim}_{D} V=\infty$, then $\left\{b(x \circ y) b^{-1}-(x \circ y)\right\}^{n}=0$ holds on $H$, the socle of $Q$, and hence it also holds on $Q$. Thus, by above Theorem 2.1, we prove the theorem in this case. So we must have $\operatorname{dim}{ }_{D} V<\infty$. Thus $Q$ is isomorphic to $D_{m}$, the $m \times m$ matrix ring over $D$ for some $m$.

If $C$ is finite, then $D$ is a finite division ring and thus is a field by Wedderburn's theorem. In this case, $Q=C_{m}$. On the other hand, if $C$ is infinite and $F$ is a 
maximal subfield of $D$, then by a Vandermonde determinant argument, we know that the condition

$$
\left\{b(x \circ y) b^{-1}-(x \circ y)\right\}^{n} \in C \quad \text { for all } x, y, \in Q .
$$

carries over to

$$
\left\{b(x \circ y) b^{-1}-(x \circ y)\right\}^{n} \in F \quad \text { for all } x, y, \in Q \otimes_{C} F .
$$

But $Q \otimes_{C} F=D_{m} \otimes F=\left(D \otimes_{C} F\right)_{m}=F_{k}$ for some $k$. In either case, we may suppose that $Q=F_{k}$ for some $k>1$. Since $\operatorname{dim}_{C} Q>4$, we see that $k>2$. Let V be a k-dimensional vector space over $F$ and $Q$ can be realized as a ring consisting of all $F$-linear transformations of $V$. For any given $v \in V$, we claim that $v$ and $v b$ are $F$-dependent. Suppose, on the contrary, that $v$ and $v b$ are $F$-independent. We reduce the proof to the following two cases.

Case 1. Assume that $v, v b, v b^{-1}$ are $F$-independent, we extend $v, v b, v b^{-1}$ to be an $F$-base $v, v b, v b^{-1}, v_{i}, \cdots v_{k}$ of ${ }_{F} V$, where $i=4,5, \cdots, k$, by the density of $Q$, there exist $x, y \in Q$ such that

$$
\begin{array}{llll}
v x=0, & v b x=v b^{-1}, & v b^{-1} x=0, & v_{i} x=0, \\
v y=0, & v b y=0, & v b^{-1} y=v b, & v_{i} y=0,
\end{array}
$$

for $i=4,5, \cdots, k$. And so

$$
v(x \circ y)=0, \quad v b(x \circ y)=v b, \quad v b^{-1}(x \circ y)=v b^{-1}, \quad v_{i}(x \circ y)=0,
$$

for $i=4,5, \cdots, k$. Hence $\left\{\left(b(x \circ y) b^{-1}\right)-(x \circ y)\right\}^{n}$ is of rank at most 2. Being in $F$, we get that $\left\{\left(b(x \circ y) b^{-1}\right)-(x \circ y)\right\}^{n}=0$. Thus we have a contradiction:

$$
0=v\left\{\left(b(x \circ y) b^{-1}\right)-(x \circ y)\right\}^{n}=v \neq 0 .
$$

Case 2. Otherwise, $v, v b, v b^{-1}$ are $F$-dependent. Since $v$ and $v b$ are $F$-independent, we extend $v, v b$ to be an $F$-base $v, v b, v_{i}, \cdots v_{k}$ of ${ }_{F} V$, where $i=3,4,5, \cdots, k$. We have $v b^{-1}=d_{1} v+d_{2} v b$, for some $d_{1}, d_{2} \in F$. Moreover, we claim that $d_{2} \neq 0$. Indeed, if $d_{2}=0$, then $v b^{-1}=d_{1} v$ and $v=d_{1} v b$, a contradiction. By the density of $Q$ there exist $x, y \in Q$ such that

$$
\begin{aligned}
& v x=v, \quad v b x=0, \quad v_{i} x=0, \\
& v y=-v, \quad v b y=v b, \quad v_{i} y=0,
\end{aligned}
$$

for $i=3,4,5, \cdots, k$. And so

$$
v(x \circ y)=-2 v, \quad v b(x \circ y)=0, \quad v_{i}(x \circ y)=0,
$$

for $i=3,4,5, \cdots, k$. Hence $\left\{\left(b(x \circ y) b^{-1}\right)-(x \circ y)\right\}^{n}$ is of rank at most 2. Being in $F$, we get that $\left\{\left(b(x \circ y) b^{-1}\right)-(x \circ y)\right\}^{n}=0$. Thus we have a contradiction:

$$
0=v\left\{\left(b(x \circ y) b^{-1}\right)-(x \circ y)\right\}^{n}=v 2^{n} \neq 0 .
$$


From the above, we have proven that $v$ and $v b$ are $F$-dependent for every $v \in$ $V$. Following the same proof as that of above theorem, we obtain that $b \in F$, a contradiction. Thus $\operatorname{dim}_{C} R C \leq 4$. In view of Lemma 2.1, $R$ satisfies $s_{4}$.

By taking a special case for $n=1$ of Theorem 2.2, we have the following corollary.

Corollary 2.1. Let $R$ be a prime ring, $I$ a nonzero ideal of $R$, and $\sigma$ a nontrivial automorphism of $R$ such that $\left\{(x \circ y)^{\sigma}-(x \circ y)\right\} \in Z$ for all $x, y \in I$, then $R$ satisfies $s_{4}$.

The following example demonstrates that $R$ to be prime is essential in Theorem 2.2 .

Example 2.1. Let $\mathbb{Z}$ be the ring of all integers. Let

$R=\left\{\left(\begin{array}{lll}0 & a & b \\ 0 & 0 & c \\ 0 & 0 & 0\end{array}\right) \mid a, b, c \in \mathbb{Z}\right\}, I=\left\{\left(\begin{array}{lll}0 & a & b \\ 0 & 0 & 0 \\ 0 & 0 & 0\end{array}\right) \mid a, b \in \mathbb{Z}\right\}$ and define $\sigma: R \rightarrow R$ by $\left(\begin{array}{ccc}0 & a & b \\ 0 & 0 & c \\ 0 & 0 & 0\end{array}\right)^{\sigma}=\left(\begin{array}{ccc}0 & -a & b \\ 0 & 0 & -c \\ 0 & 0 & 0\end{array}\right)$. Then $I$ is a nonzero ideal of $R$, and it is easy to see that $\sigma$ is an automorphism of $R$ such that $\left\{(x \circ y)^{\sigma}-(x \circ y)\right\}^{n} \in Z$ for all $x, y \in I$, but $R$ is not commutative. Hence, in theorem the hypothesis of primeness cannot be omitted.

\section{References}

[1] N. Argac, H. G. Inceboz, Derivation of prime ring and semiprime rings, J. Korean Math. Soc., 46(5)(2009), 997-1005.

[2] M. Ashraf, N. Rehman, On commutativity of rings with derivations, Results Math., 42(1-2)(2002), 3-8.

[3] K. I. Beidar, W. S. Martindale III, A. V. Mikhalev, Rings with Generalized Identities, New York-Basel-Hong Kong: Marcel Dekker, Inc., (1996), 51-95.

[4] H. E. Bell, W. S. Martindale, Centralizing mappings of semiprime rings, Can. Math. Bull., 30(1987), 92-101.

[5] M. Brešar, centralizing mappings and derivations in prime rings, J. Algebra, 156(1993), 385-394.

[6] M. Brešar, On skew commuting mappings of rings, Bull. Aust. Math. Soc., 47(1993), 291-296.

[7] M. Brešar, Functional identities: a survey, Contemporary Math., 259(2000), 93-109.

[8] C. L. Chuang, GPIs having coefficients in Utumi quotient rings, Proc. Amer. Math. Soc., 103(3)(1988), 723-728,. 
[9] C. L. Chuang, Differential identities with automorphisms and antiautomorphisms II, J. Algebra, 160(1993), 130-171.

[10] N. Divinsky, On commuting automorphisms of rings, Trans. R. Soc. Can. Sect. III, 49(1955), 19-22.

[11] J. S. Erickson, W. S. Martindale, J. M. Osborn, Prime nonassociative algebras, Pacific J. Math., 60(1975), 49-63.

[12] N. Jacobson, PI-algebras: An Introduction, Lecture Notes in Mathematics, Vol. 441. Berlin HeidelbergNew York: Springer Verlag, (1975).

[13] C. Lanski, Left ideals and derivations in semiprime ring, J. Algebra, 277(2004), 658667.

[14] J. Luh, A note on commuting automorphisms of rings, Amer. Math. Mont., 77(1970), 61-62.

[15] W. S. Martindale, Prime rings satisfying a generalized polynomial identity, J. Algebra, 12(1969), 576-584.

[16] J. H. Mayne, Centralizing automorphisms of prime ring, Canad. Math. Bull., 19(1976), 113-115.

[17] E. C. Posner, Derivations in prime rings, Proc. Amer. Math. Soc., 8(1957), 10931100.

[18] Y. Wang, Power-centralizing automorphisms of Lie ideals in prime ring, Comm. Algebra, 34(2006), 609-615. 